\title{
Parental Shading Regulates Subsequent Seed Germination
}

\author{
Lei Wang ${ }^{1,2+}$, Umashankar Chandrasekaran ${ }^{1 \dagger}$, Xiaofeng Luo ${ }^{1}$, Shaowei Wei ${ }^{1}$ and \\ Kai Shu ${ }^{1,2 * t}$ \\ ${ }^{1}$ School of Ecology and Environment, Northwestern Polytechnical University, Xi'an, China, ${ }^{2}$ Research \& Development \\ Institute of Northwestern Polytechnical University in Shenzhen, Shenzhen, China
}

Keywords: gibberellic acid, abscisic acid, seed germination, phytohormone, parental shading

\section{INTRODUCTION}

Seed germination is essential for subsequent young seedling establishment. Numerous elegant studies have documented the regulatory mechanisms underlying seed germination, especially phytohormones and environmental cues-mediated cascades (Shu et al., 2016; Tognacca and Botto, 2021). The promotion effect of phytohormone abscisic acid (ABA) and the repression effect of

Edited by:

$\mathrm{Lin} \mathrm{Li}$,

Fudan University, China

Reviewed by:

Hui Shi,

Capital Normal University, China

Yi TaO,

Xiamen University, China

*Correspondence:

Kai Shu

kshu@nwpu.edu.cn orcid.org/0000-0003-4888-3001

tThese authors have contributed equally to this work

Specialty section:

This article was submitted to

Plant Physiology,

a section of the journal

Frontiers in Plant Science

Received: 28 July 2021

Accepted: 12 October 2021

Published: 08 November 2021

Citation:

Wang L, Chandrasekaran U, Luo X,

Wei S and Shu K (2021) Parental

Shading Regulates Subsequent Seed

Germination.

Front. Plant Sci. 12:748760.

doi: 10.3389/fp/s.2021.748760 gibberellin (GA) on seed germination are extensively detected and well-documented (Shu et al., 2016, 2018). Furthermore, diverse environmental factors are also involved in seed germination control, including temperature, light, salinity, and drought. It is noted that most research about seed germination is mainly focused on the roles of these endogenous and/or environmental cues specifically during the seed imbibition stage (Shu et al., 2013, 2016; Luo et al., 2021). Whereas few studies into the effects of exposure of the parental plants to some environmental cues on subsequent seed germination processes have been published.

Among the diverse environmental factors influencing seed germination, light is attractive, which not only acts as an energy resource, but also the molecular signal for initiating seed germination (Wang and Lin, 2020). It is known that close planting leads plants to perceive the shade signal, characterized with the decrease of blue light intensity and the red (R): far-red (FR) ratio, caused by neighboring plants (Keuskamp et al., 2011, 2012; de Wit et al., 2012; Jiang et al., 2019; Zhang et al., 2019; Yang et al., 2020). In close planting conditions, competition for light triggers the plant shade response, including the change of flowering time, promotion of stem and petiole elongation, regulation of seed maturation, variation in photosynthetic response, and decrease of crop productivity (Kurepin et al., 2006; Jha et al., 2010; Elwell et al., 2011; Baker et al., 2018; Chai et al., 2018; Pantazopoulou et al., 2021). Thus, plant shade response plays a key role during the plant life cycle, and is especially important for modern agricultural production. In this research field, the effect of shade signaling on seed development and dormancy/germination are interesting and worthwhile projects, and recently some progress has been achieved. For instance, our previous study showed a higher germination rate in seeds developed under shade conditions compared to the control group, by mediating the biosynthesis of pro-anthocyanidins, fatty acids, and phytohormones ABA and GA (Chen et al., 2020). Indeed, except for the shading signal (Contreras et al., 2008), other maternal environmental cues, such as temperature, are also involved in subsequent seed dormancy and germination regulation (Kvaalen and Johnsen, 2008; Postma and Agren, 2015). Here, we concisely summarized the current understanding of parental shademeditated seed biology, focusing on the regulatory roles of parental shading in subsequent seed dormancy and germination. 


\section{PARENTAL SHADING MODULATES PHYTOHORMONE BALANCE IN DEVELOPING SEEDS}

There is a large amount of evidence reporting that diversity in phytohormones is involved in plant shade response (Sellaro et al., 2012; Sessa et al., 2018). Arabidopsis seeds under shade conditions show a reduction in germination with a significant increase in $\mathrm{ABA}$ and 12-oxo-phytodienoic acid (OPDA) content (BarrosGalvão et al., 2019). Further, an earlier study showed that seeds matured under FR light have an increased thermo-inhibition and photo-sensing capacity as well as ABA level, which in turn affect subsequent seed germination (Contreras et al., 2009). Blue light receptor CRYTOCHROME1 (CRY1) enhances seed dormancy by increasing the accumulation of $\mathrm{ABA}$ in Arabidopsis seeds under blue light-rich conditions (Barrero et al., 2014). These studies highlighted the important effect of parental shading on subsequent seed dormancy and germination control.

$R$ light enhances seed germination through inhibition of the expression of ABA biosynthesis genes, while FR light delays seed germination by promoting the transcription of ABA biosynthesis genes (Barrero et al., 2014). Our previous study demonstrated that soybean seeds matured under parental shading show an increased germination rate, supported by an increase in endogenous GA content and a decrease in ABA levels, and consistently the expression level of genes involved in ABA biosynthesis are downregulated in shade condition grown seeds, while the transcription levels of the genes related to GA biosynthesis are upregulated (Chen et al., 2020). Thus, the balance between $\mathrm{ABA}$ and $\mathrm{GA}$ in regulating seed germination after shading treatment is significant (Figure 1). However, a detailed molecular analysis of the balance between ABA and GA in the seeds matured under shade conditions is currently unknown. For instance, how does shade signal regulate the corresponding genes expression? What are the key transcription factors involved in this cascade? More importantly, how the ABA-dependent primary seed dormancy is released in seeds subjected to shade is a worthwhile project to be addressed.

It is interesting that the distinct effects of parental shading on subsequent seed germination in different species are documented. For instance, in lettuce, parental shading has a negative role in subsequent seed germination (Contreras et al., 2009), while the promotion effect of parental shading on seed germination was detected in soybean (Chen et al., 2020). The difference of seed storage proteins, seed size, or even evolutionary history between lettuce and soybean may cause the distinct responsiveness for parental shading signal during seed germination, but the underlying molecular mechanisms need to be further explored.

In addition to the research on cultivated crops such as soybean (Chen et al., 2020), the similar effect of parental shading on seed size and yield in native wild species has also been detected. A recent study showed that, in Primula vulgaris, shading in the maternal environment led to increased seed size, but the effect of parental shading on subsequent seed germination is weak (Marin et al., 2019). Except for the seed germination processes, young

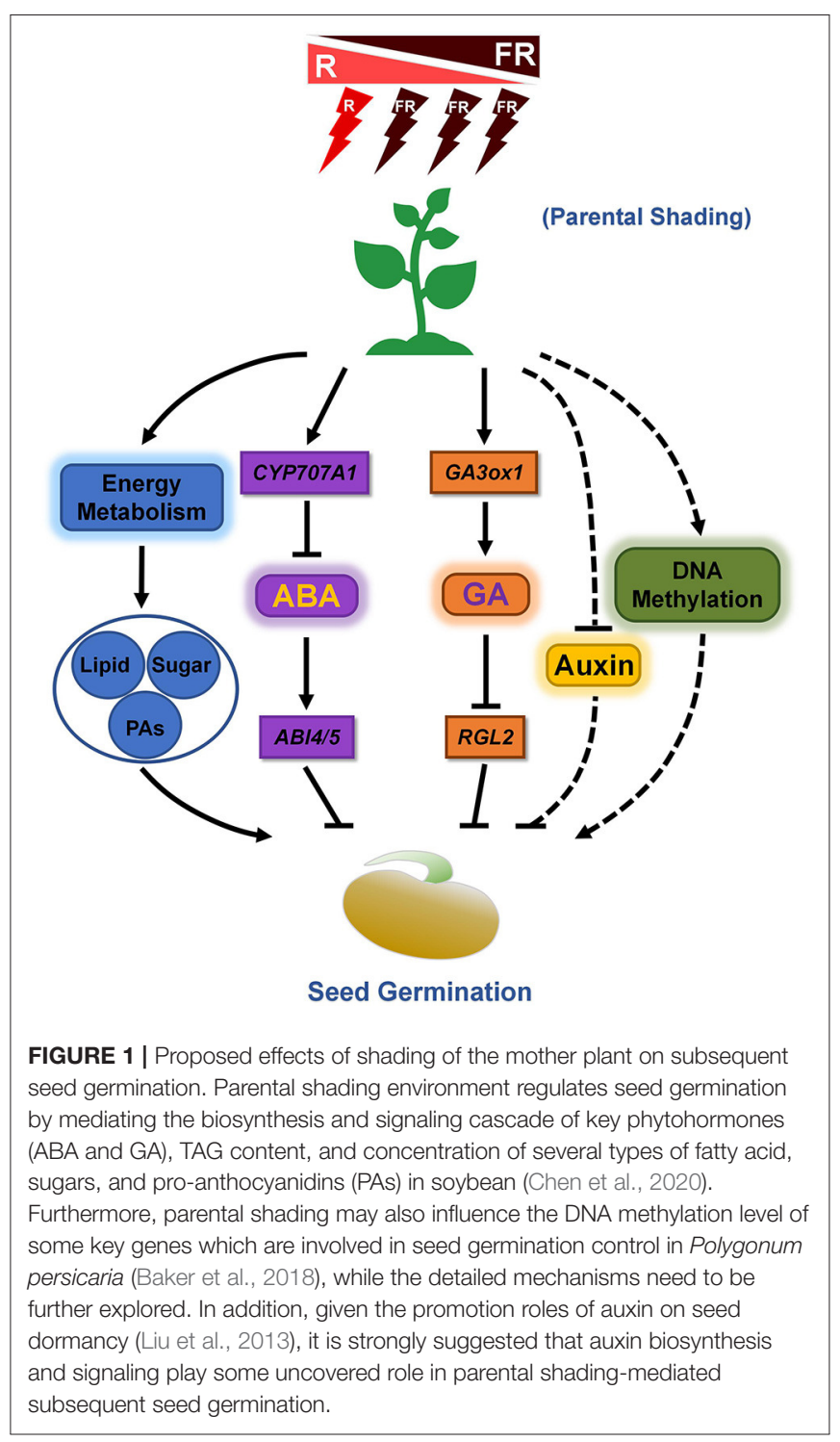

seedling establishment and development are also regulated by the parental shade environment probably by mediating DNA methylation modification (Baker et al., 2018), indicating that DNA methylation regulates transgenerational environmental effects between parents and offspring (Figure 1). However, the detailed epigenetic regulatory mechanisms especially underlying phytohormones-relevant information need to be further dissected.

\section{PARENTAL SHADING REGULATES STORAGE ENERGY RESOURCES IN DEVELOPING SEEDS}

Seed germination is driven by the energy stored in the seed itself (Eastmond, 2004), and especially in oil-containing seeds, such as oil rape seeds, soybean, and Arabidopsis seeds, the hydrolysis of 
triacylglycerol releases glycerol and fatty acids, and then the latter are converted to sugars which fuels seed germination (Eastmond, 2006; Bachleda et al., 2017; Zhou et al., 2019). Therefore, the levels of several types of energy resources in seeds, including sugars and fatty acid, are important for seed germination processes.

A previous study demonstrated that parental shading environment influenced the concentrations of several types of sugars and fatty acids during soybean seed development, and some of which are known to be associated with seed germination regulation. For instance, the concentrations of oleic and linolenic acid decreased in shaded-development seeds, while the concentration of linoleic acid increased, which is consistent with the faster germination phenotype of shaded-development seeds (Chen et al., 2020). Furthermore, given the repression effects of pro-anthocyanidins in seed germination (Jia et al., 2012), parental shading treatment also downregulated the levels of soluble pro-anthocyanidins in developing seeds, which further enhanced subsequent seed germination (Chen et al., 2020). Thus, the shade environment of the parent plants affects the concentration of soluble pro-anthocyanidins and several types of sugars as well as fatty acids, and finally control subsequent seed germination (Figure 1).

\section{FUTURE PROSPECTS}

Perception and signaling of environmental changes are essential during the plant life cycle, including seed development and germination periods. Despite the abundance of the effect of shading on plant growth, information regarding the influence of parental shading on seed maturation and subsequent seed dormancy and germination are still elusive.

The effects of parental shading on the levels of ABA and GA in developing seeds were documented (Chen et al., 2020). It is noted that the other important phytohormone auxin plays key roles in seed dormancy and germination control (Liu et al., 2013), thus the molecular mechanisms of auxin in regulating seed germination after parental shading need to be further explored. Especially, investigation on auxin transport, signaling, and homeostasis during seed development under shading might dissect several unknown cascades beyond ABA and GA-mediated pathways (Figure 1).

\section{REFERENCES}

Bachleda, N., Grey, T., and Li, Z. L. (2017). Effects of high oleic acid soybean on seed yield, protein and oil contents, and seed germination revealed by near-isogeneic lines. Plant Breed. 136, 539-547. doi: 10.1111/pbr.12497

Baker, B. H., Berg, L. J., and Sultan, S. E. (2018). Context-dependent developmental effects of parental shade versus sun are mediated by DNA methylation. Front. Plant Sci. 9, 1251-1251. doi: 10.3389/fpls.2018.01251

Barrero, J. M., Downie, A. B., Xu, Q., and Gubler, F. (2014). A role for barley CRYPTOCHROME1 in light regulation of grain dormancy and germination. Plant Cell 26, 1094-1104. doi: 10.1105/tpc.113.121830

Barros-Galvão, T., Dave, A., Cole, A., Harvey, D., Langer, S., Larson, T. R., et al. (2019). cis-12-Oxo-phytodienoic acid represses Arabidopsis seed germination in shade conditions. J. Exp. Bot. 70, 5919-5927. doi: 10.1093/jxb/erz337
Under parental shading conditions, what and how do the multiple photoreceptors (especially including phytochromes, cryptochromes, and phototropins) regulate seed development, subsequent seed dormancy release, and seed germination processes? Further, what are the relationships between the photoreceptors and phytohormones biosynthesis/signaling cascades under the parental shading environment? Indeed, these studies focusing on the parental effect on subsequent offspring growth and development provide good case studies for investigating cross-generational effects in plants induced by environmental cues. More importantly, we hope that the underlying genetic mechanisms using epigenetic approaches, including genomic DNA methylation and other molecular effects, and the precise mechanisms underlying the positive effect of parental shade signals on subsequent seed germination will be uncovered in the near future. The outcome of the proposed research ideas will provide valuable information to engineer seeds with resisting capacity under unfavorable environmental conditions without affecting the crop productivity.

\section{AUTHOR CONTRIBUTIONS}

LW, UC, and KS designed and jointly wrote this Opinion article. XL and SW provided inputs for the improvement of the article. All authors contributed to the article and approved the submitted version.

\section{FUNDING}

Funding from the National Natural Science Foundation of China (32101670), the Science, Technology, and Innovation Commission of Shenzhen Municipality (JCYJ20190806154009040), and the Talents Team Construction Fund of Northwestern Polytechnical University (31020190QD007) supported the work in our group.

\section{ACKNOWLEDGMENTS}

We thank all members in the lab for the discussion during the preparation of this manuscript. We also apologize to all colleagues whose work could not be discussed and cited because of limited space.

Chai, S., Tang, J., Mallik, A., Shi, Y., Zou, R., Li, J., et al. (2018). Ecophysiological basis of shade adaptation of Camellia nitidissima, a rare and endangered forest understory plant of Southeast Asia. BMC Ecol. 18:5. doi: 10.1186/s12898-018-0159-y

Chen, F., Zhou, W., Yin, H., Luo, X., Chen, W., Liu, X., et al. (2020). Shading in mother plant during seed development promotes subsequent seed germination in soybean. J. Exp. Bot. 71, 2072-2084. doi: 10.1093/jxb/erz553

Contreras, S., Bennett, M., Metzger, J., and Tay, D. (2008). Maternal light environment during seed development affects lettuce seed weight, germinability, and storability. Hortscience 43, 845-852. doi: 10.21273/HORTSCI.43.3.845

Contreras, S., Bennett, M., Metzger, J., Tay, D., and Nerson, H. (2009). Red to far-red ratio during seed development affects lettuce seed germinability and longevity. Hortscience 44, 130-134. doi: 10.21273/HORTSCI.44.1.130 
de Wit, M., Kegge, W., Evers, J. B., Vergeer-van Eijk, M. H., Gankema, P., Voesenek, L. A. C. J., et al. (2012). Plant neighbor detection through touching leaf tips precedes phytochrome signals. Proc. Natl. Acad. Sci. 109:14705. doi: 10.1073/pnas.1205437109

Eastmond, P. J. (2004). Glycerol-insensitive Arabidopsis mutants: gli1 seedlings lack glycerol kinase, accumulate glycerol and are more resistant to abiotic stress. Plant J. 37, 617-625. doi: 10.1111/j.1365-313X.2003.01989.x

Eastmond, P. J. (2006). SUGAR-DEPENDENT1 encodes a patatin domain triacylglycerol lipase that initiates storage oil breakdown in germinating Arabidopsis seeds. Plant Cell 18, 665-675. doi: 10.1105/tpc.105.040543

Elwell, A. L., Gronwall, D. S., Miller, N. D., Spalding, E. P., and Durham Brooks, T. L. (2011). Separating parental environment from seed size effects on next generation growth and development in Arabidopsis. Plant Cell Environ. 34, 291-301. doi: 10.1111/j.1365-3040.2010.02243.x

Jha, P., Norsworthy, J. K., Riley, M. B., and Bridges, W. (2010). Shade and plant location effects on germination and hormone content of palmer amaranth (Amaranthus palmeri) seed. Weed Sci. 58, 16-21. doi: 10.1614/WS-09-059.1

Jia, L. G., Wu, Q. Y., Ye, N. H., Liu, R., Shi, L., Xu, W. F., et al. (2012). Proanthocyanidins inhibit seed germination by maintaining a high level of abscisic acid in Arabidopsis thaliana. J. Integr. Plant Biol. 54, 663-673. doi: 10.1111/j.1744-7909.2012.01142.X

Jiang, Y., Yang, C., Huang, S., Xie, F., Xu, Y., Liu, C., et al. (2019). The ELF3-PIF7 interaction mediates the circadian gating of the shade response in Arabidopsis. iScience 22, 288-298. doi: 10.1016/j.isci.2019.11.029

Keuskamp, D. H., Keller, M. M., Ballaré, C. L., and Pierik, R. (2012). Blue light regulated shade avoidance. Plant Signal. Behav. 7, 514-517. doi: $10.4161 /$ psb.19340

Keuskamp, D. H., Sasidharan, R., Vos, I., Peeters, A. J. M., Voesenek, L. A. C. J., and Pierik, R. (2011). Blue-light-mediated shade avoidance requires combined auxin and brassinosteroid action in Arabidopsis seedlings. Plant J. 67, 208-217. doi: 10.1111/j.1365-313X.2011.04597.x

Kurepin, L. V., Pharis, R. P., Reid, D. M., and Chinnappa, C. C. (2006). Involvement of gibberellins in the stem elongation of sun and shade ecotypes of Stellaria longipes that is induced by low light irradiance. Plant Cell Environ. 29, 1319-1328. doi: 10.1111/j.1365-3040.2006.01512.x

Kvaalen, H., and Johnsen, O. (2008). Timing of bud set in Picea abies is regulated by a memory of temperature during zygotic and somatic embryogenesis. New Phytol. 177, 49-59. doi: 10.1111/j.1469-8137.2007.02222.x

Liu, X., Zhang, H., Zhao, Y., Feng, Z., Li, Q., Yang, H. Q., et al. (2013). Auxin controls seed dormancy through stimulation of abscisic acid signaling by inducing ARF-mediated ABI3 activation in Arabidopsis. Proc. Natl. Acad. Sci. U. S. A. 110, 15485-15490. doi: 10.1073/pnas.1304651110

Luo, X., Dai, Y., Zheng, C., Yang, Y., Chen, W., Wang, Q., et al. (2021). The ABI4RbohD/VTC2 regulatory module promotes reactive oxygen species (ROS) accumulation to decrease seed germination under salinity stress. New Phytol. 229, 950-962. doi: 10.1111/nph.16921

Marin, M., Blandino, C., Laverack, G., Toorop, P., and Powell, A. A. (2019). Responses of Primula vulgaris to light quality in the maternal and germination environments. Plant Biol. (Stuttg) 21, 439-448. doi: 10.1111/plb.12849

Pantazopoulou, C. K., Bongers, F. J., and Pierik, R. (2021). Reducing shade avoidance can improve Arabidopsis canopy performance against competitors. Plant Cell Environ. 44, 1130-1141. doi: 10.1111/pce.13905

Postma, F. M., and Agren, J. (2015). Maternal environment affects the genetic basis of seed dormancy in Arabidopsis thaliana. Mol. Ecol. 24, 785-797. doi: $10.1111 / \mathrm{mec} .13061$
Sellaro, R., Pacín, M., and Casal, J. J. (2012). Diurnal dependence of growth responses to shade in Arabidopsis: role of hormone, clock, and light signaling. Mol. Plant 5, 619-628. doi: 10.1093/mp/ssr122

Sessa, G., Carabelli, M., Possenti, M., Morelli, G., and Ruberti, I. (2018). Multiple pathways in the control of the shade avoidance response. Plants 7:102. doi: 10.3390/plants7040102

Shu, K., Liu, X. D., Xie, Q., and He, Z. H. (2016). Two faces of one seed: hormonal regulation of dormancy and germination. Mol. Plant 9, 34-45. doi: 10.1016/j.molp.2015.08.010

Shu, K., Zhang, H., Wang, S., Chen, M., Wu, Y., Tang, S., et al. (2013). ABI4 regulates primary seed dormancy by regulating the biogenesis of abscisic acid and gibberellins in arabidopsis. PLoS Genet. 9:e1003577. doi: 10.1371/journal.pgen.1003577

Shu, K., Zhou, W., Chen, F., Luo, X., and Yang, W. (2018). Abscisic acid and gibberellins antagonistically mediate plant development and abiotic stress responses. Front. Plant Sci. 9:416. doi: 10.3389/fpls.2018. 00416

Tognacca, R. S., and Botto, J. F. (2021). Post-transcriptional regulation of seed dormancy and germination: current understanding and future directions. Plant Commun. 2:100169. doi: 10.1016/j.xplc.2021.100169

Wang, Q., and Lin, C. (2020). Mechanisms of cryptochrome-mediated photoresponses in plants. Annu. Rev. Plant Biol. 71, 103-129. doi: 10.1146/annurev-arplant-050718-100300

Yang, F., Liu, Q., Cheng, Y., Feng, L., Wu, X., Fan, Y., et al. (2020). Low red/far-red ratio as a signal promotes carbon assimilation of soybean seedlings by increasing the photosynthetic capacity. BMC Plant Biol. 20:148. doi: 10.1186/s12870-020-02352-0

Zhang, R., Yang, C., Jiang, Y., and Li, L. (2019). A PIF7-CONSTANScentered molecular regulatory network underlying shade-accelerated flowering. Mol. Plant 12, 1587-1597. doi: 10.1016/j.molp.2019.0 9.007

Zhou, W. G., Chen, F., Zhao, S. H., Yang, C. Q., Meng, Y. J., Shuai, H. W., et al. (2019). DA-6 promotes germination and seedling establishment from aged soybean seeds by mediating fatty acid metabolism and glycometabolism. J. Exp. Bot. 70, 101-114. doi: 10.1093/jxb/er y 247

Conflict of Interest: The authors declare that the research was conducted in the absence of any commercial or financial relationships that could be construed as a potential conflict of interest.

Publisher's Note: All claims expressed in this article are solely those of the authors and do not necessarily represent those of their affiliated organizations, or those of the publisher, the editors and the reviewers. Any product that may be evaluated in this article, or claim that may be made by its manufacturer, is not guaranteed or endorsed by the publisher.

Copyright $(2021$ Wang, Chandrasekaran, Luo, Wei and Shu. This is an open-access article distributed under the terms of the Creative Commons Attribution License (CC $B Y)$. The use, distribution or reproduction in other forums is permitted, provided the original author(s) and the copyright owner(s) are credited and that the original publication in this journal is cited, in accordance with accepted academic practice. No use, distribution or reproduction is permitted which does not comply with these terms. 